\title{
Configurational Entropy of Optical Bright Similariton in Tapered Graded-Index Waveguide
}

\author{
Pooja Thakur ${ }^{\mathrm{a}}$, Marcelo Gleiser ${ }^{\mathrm{b}}$, Anil Kumar ${ }^{\mathrm{c}}$, Rama Gupta ${ }^{\mathrm{a}}$ \\ ${ }^{a}$ Department of Physics, D. A. V. University, Jalandhar-144 012, Punjab, India \\ ${ }^{b}$ Department of Physics and Astronomy, Darmouth College, Hanover, New Hampshire \\ 03755, USA \\ ${ }^{c}$ Department of Physics, JC DAV College (Panjab University), Dasuya-144 205, Punjab, \\ India
}

\begin{abstract}
Configurational entropy (CE) consists of a family of entropic measures of information used to describe the shape complexity of spatially-localized functions with respect to a set of parameters. We obtain the Differential Configurational Entropy (DCE) for similariton waves traveling in tapered gradedindex optical waveguides modeled by a generalized nonlinear Schrödinger equation. It is found that for similariton's widths lying within a certain range, DCE attains minimum saturation values as the nonlinear wave evolves along the effective propagation variable $\zeta(t)$. In particular, saturation is achieved earlier for lower values of the width, which we show correspond to global minima of the DCE. Such low entropic values lead to minimum dispersion of momentum modes as the similariton waves propagate along tapered gradedindex waveguides, and should be of importance in guiding their design.
\end{abstract}

Keywords: Configurational Entropy, Tapered Graded-Index Waveguide, Optical Fiber Communications, Generalized Nonlinear Schrödinger

Equation, Bright Similariton.

PACS: code 49.70. +c, 42.65. Wi, 42.65.Tg, 05.90.+m.

Email addresses: poojaphy19@gmail.com (Pooja Thakur), mgleiser@dartmouth.edu (Marcelo Gleiser), anilkumarphys@gmail.com (Anil Kumar), Corresponding author:rama.gupta@gmail.com (Rama Gupta) 


\section{Introduction}

Nonlinear phenomena appear in all areas of science, from physics and chemistry to the life sciences and engineering [1, 2]. In most applications, nonlinear systems are modelled by partial differential equations called nonlinear evolution equations (NLEEs). Of much interest are solutions in the shape of spatially-localized excitations. Many such excitations are non-dispersive and non-dissipative waves, that is, configurations that are weakly or nonradiative and that maintain their shape as they propagate to significantly long distances [3]. Due to this special feature, these configurations are known as solitons or solitary waves. Soliton-like solutions appear in various research areas, including hydrodynamics, plasma physics, nonlinear optics, condensed matter physics, optical communications, nuclear physics, and astrophysics [4, 5, 6, 7, 8, 9].

Different strategies are used to investigate the dynamical features of soliton-like solutions from NLEEs, including exact analytical methods [10], numerical techniques [11], variational analysis, and other tools [12]. A new emerging area is the study of the configurational entropy (CE) of these nonlinear excitations, a measure of spatial complexity of spatially-localized systems proposed by Gleiser and Stamatopoulos, originally applied to the study of kinks and bounces in relativistic field theory [14]. As described in detail in Ref. [23], the original CE has been expanded to include a family of related entropic measures, Configuration Information Measures (CIMs), adapted for different applications. In the current case, for a continuum field theory, the proper measure is known as Differential Configurational Entropy (DCE), as defined below. It gives a quantitative measure of the shape complexity of a function or, in the case of most physical applications, of a given field configuration.

Inspired by Shannon information entropy [13], the DCE is obtained from the Fourier transform of a spatially-localized configuration (e.g. a solution of a NLEE) or its related energy density. There is an intimate link between information and dynamics, where the entropic measure plays a prominent role in signaling the emergence of nonlinear structures and in highlighting their stability properties [14, 15]. Configurational Entropy has since been applied to a variety of fields, including neutron and boson stars [16], spontaneous symmetry breaking [15], glueballs [17], the stability of Q-balls [18], anti-de Sitter black holes [19, 20], stability of graviton Bose-Einstein condensate in the brane-world [21], the energy-energy correlation in $e^{+} e^{-}$into 
hadrons [22], oscillon lifetimes in scalar field theories [23], instantons and vacuum decay in arbitrary spatial dimensions [24], dynamical tachyonic holographic Ads/QCD models [25], standard model cosmology for the homogeneous Friedmann-Robertson-Walker universe [26] and in inflationary cosmology [27], and in the study of dissociation of heavy vector mesons in a thermal medium [28]. Apart from the above applications in high-energy physics and cosmology, DCE has been calculated for several nonlinear scalar field models featuring solutions with spatially-localized energy, including bounces in one spatial dimension and critical bubbles in three spatial dimensions [14], for the determination of critical points in second order phase transitions [29], solitons in supersymmetric theories [30], and for Korteweg-de Vries solitons in quark-gluon plasmas [31].

In the present work, we apply the DCE measure to solitary waves propagating along tapered graded-index nonlinear waveguides. The graded potential is widely used in optical fiber communications [32], computer networks [33], long-distance telecommunications [34], and sensory receptor cells [35]. It solves the problem of modal dispersion to a considerable extent [33]. In particular, we will investigate the propagation of waves in tapered gradedindex waveguides modeled by a generalized nonlinear Schrödinger equation (GNLSE) [36, 37, 38] and obtain the DCE for an optical similariton, a solitonic solution of the GNLSE with variable coefficient.

The paper is organized as follows: In section 2, we briefly review DCE. (For a more detailed discussion see Ref. [23].) In section 3, the similariton solution of GNLSE is obtained invoking the similarity transformation, as well as the saturation minimum-value DCE as a function of width as the similariton evolves in time. We also find that the similariton's DCE has global minima as a function of width. These time-dependent values determine the optimal value of the width for the configurational stability of similaritons propagating through tapered graded-index optical waveguides. Finally, we summarize our results in section 4 .

\section{Differential Configurational Entropy for bright similaritons in tapered graded-index waveguide}

The Differential Configuration Entropy of a square-integrable, bounded mathematical function is constructed from its Fourier modes [14, 23]. In physical applications, it is often the case that the square-integrable continuous func- 
tion is the energy density $\rho(x)$ defined on $R^{d}$ and with Fourier transform,

$$
F(k)=\int \exp [-i x \cdot k] \rho(x) d^{d} x
$$

Henceforth, we consider only $d=1$. The corresponding modal fraction, which measures the relative weight of each mode $k$ is defined as [14]

$$
f(k)=\frac{|F(k)|^{2}}{\int|F(k)|^{2} d k} .
$$

To ensure positivity of DCE, the modal fraction is normalized by the mode carrying maximum weight $f_{\max }(k)$,

$$
\tilde{f}(k)=\frac{f(k)}{f_{\max }(k)} .
$$

The mathematical expression of DCE is defined as $S_{c}[\tilde{f}]$ is $[14]$

$$
S_{c}[\tilde{f}]=-\int_{-\infty}^{\infty} \tilde{f}(k) \ln \tilde{f}(k) d k,
$$

which represents an absolute limit on the best lossless compression of any communication [39]. For periodic functions, one would use the Fourier series of the function $\rho(x)$ to define DCE. While other possible functional transforms could in principle be used to obtain the DCE, the clear physical interpretation of Fourier transform relating increased spatial localization to broader momentum-mode distribution, makes it the most efficient to define DCE, as the many applications referenced above have shown.

\section{Differential Configurational Entropy for bright similaritons in tapered graded-index waveguide}

\subsection{Model equation and similariton solution}

The beam propagation in tapered graded-index nonlinear fiber waveguide is modelled by the GNLSE [36, 37, 38]. The GNLSE in $(1+1)$-dimensional form is given as

$$
i \frac{\partial \Psi}{\partial t}+\frac{1}{2} a^{2}(t) \frac{\partial^{2} \Psi}{\partial x^{2}}+\frac{1}{2} M(t) x \Psi-\frac{i}{2} g(t) \Psi+2 \gamma(t) \Psi+\mu|\Psi|^{2} \Psi=0 .
$$


Here $a(t), M(t)$, and $\gamma(t)$ are the time-dependent dispersion coefficient, tapered potential, and external potential, respectively, while $x$ is the transverse direction and $\Psi$ is the wave function. Further, $g(t)$ is a dimensionless net energy gain (if $g>0$ ) or loss (if $g<0$ ) in the system, and $\mu$ is the coefficient of nonlinearity.

Introducing the similarity variable $\chi$,

$$
\chi(x, t)=\frac{\left[x-x_{c}(t)\right]}{\alpha(t)},
$$

the self-similar optical similariton solution of Eq. (5) can be obtained by transforming it into a standard nonlinear Schrödinger equation (NLSE) by using gauge and similarity transformations [36, 37, 38]

$$
\Psi(x, t)=B(t) \Phi[\chi(x, t), \zeta(t)] \exp [i \varphi(x, t)],
$$

where $B(t), \alpha(t)$, and $x_{c}(t)$ are the dimensionless amplitude, width, and guiding-center coordinate of the beam, respectively.

Assuming a linear ansatz for the global phase,

$$
\varphi(x, t)=\left[p_{1}(t) x+p_{2}(t)\right],
$$

and substituting Eqs. (7) and (8) into Eq. (5), we obtain a set of first-order differential equations for the parameters of the transformation and for the transformed field $\Phi$, which satisfies the standard NLSE [40]

$$
i \frac{\partial \Phi}{\partial \zeta}+\frac{1}{2} \frac{\partial^{2} \Phi}{\partial \chi^{2}}+\mu|\Phi|^{2} \Phi=0
$$

Here, the effective propagation variable $\zeta(t)$ and guiding-center position $x_{c}(t)$ are given by

$$
\begin{gathered}
\zeta(t)=\zeta_{0}+\int_{0}^{t} B^{2}(\tau) d \tau \\
x_{c}(t)=\alpha(t)\left[\int_{0}^{t} \frac{a^{2}(\tau) p_{1}(\tau) d \tau}{\alpha(\tau)}+x_{0}\right],
\end{gathered}
$$

where $\zeta_{0}$ and $x_{0}$ are integration constants which we fix as $\zeta_{0}=1, x_{0}=1$. The remaining nonlinear partial differential equations for the parameters are

$$
B(t)=\frac{a(t)}{\alpha},
$$




$$
\begin{gathered}
g(t)=2 \frac{\dot{B}(t)}{B(t)}, \\
M(t)=2 \dot{p}_{1}(t), \\
\gamma(t)=\frac{1}{2} \dot{p}_{1}(t)+a^{2}(t) p_{1}^{2}(t) .
\end{gathered}
$$

Eq. (9) is a standard NLSE, admitting bright [40] and dark [41] soliton solutions, as well as rogue waves and breathers [42]. Using these, and width $\alpha(t)$ constant, one can obtain solutions of Eq. (5) implementing the self-similar transformation of Eq. (77). In particular, the bright similariton solution of Eq. (91) is given as

$$
\Psi(x, \zeta(t))=a\left(\sqrt{\frac{\mu^{3}}{2}} \operatorname{sech}\left(\mu^{2} \frac{\left(\sqrt{2}\left(\frac{x-x_{c}}{\alpha}\right)-\chi_{0}\right)}{2}+2 \xi \zeta \mu^{2}\right) \exp \left(r_{1}\right)\right) r_{0},
$$

where

$$
\begin{gathered}
r_{0}=\exp \left(i\left(p_{1} \cdot x+p_{2}\right)\right), \\
r_{1}=-4 i\left(\xi^{2}-\left(\frac{\mu^{2}}{4}\right)^{2}\right) \zeta-2 i \xi \sqrt{2}\left(\frac{x-x_{c}}{\alpha}\right)+i \epsilon .
\end{gathered}
$$

In Eq. (12), the dispersion coefficient $a(t)$ is the amplitude of the similariton, which, in general, could have space and time dependence. Here, we have chosen it to be a periodic function of $t$,

$$
a(t)=\frac{1+\cos ^{2}(t)}{\alpha} .
$$

\subsection{Calculation of Differential Configurational Entropy}

The energy density corresponding to the solution (Eq. (12)) can be written as

$$
\rho(x, \zeta(t))=\frac{\mu^{2} \operatorname{sech}\left[2 \mu^{2} \zeta \xi+\frac{1}{2} \mu^{2}\left(\frac{\sqrt{2}\left(x-x_{c}\right)}{\alpha}-\chi_{0}\right)\right]^{2}}{2 \sqrt{2} \alpha} .
$$

The square of the Fourier transform of the energy density $F^{2}(k)$, using Eq.(11), is given as 


$$
F^{2}(k, \zeta(t))=\frac{r_{2}\left(\frac{-i r_{3}\left(-i k \alpha+\sqrt{2} \mu^{2}\right)}{r_{3}+r_{4}}+k \alpha r_{5}\right)\left(\frac{i r_{3}\left(i k \alpha+\sqrt{2} \mu^{2}\right)}{r_{3}+r_{4}}+k \alpha r_{7}\right)}{\pi\left(k^{2} \alpha^{2}+2 \mu^{2}\right)}
$$

where

$$
\begin{gathered}
r_{2}=\frac{\exp \left(-2 \mu^{2}\left(\sqrt{2} x_{c}+\alpha\left(-4 \zeta \xi+\chi_{0}\right)\right)\right)}{\alpha}, \\
r_{3}=\frac{\exp \left(\mu^{2}\left(\sqrt{2} x_{c}+\alpha \chi_{0}\right)\right)}{\alpha}, \\
r_{4}=\exp \left(4 \zeta \xi \mu^{2}\right) \\
r_{5}=2 F 1\left[1,1-\frac{i k \alpha}{\sqrt{2} \mu^{2}}, 2-\frac{i k \alpha}{\sqrt{2} \mu^{2}}, r_{6}\right] \\
r_{6}=\frac{\exp \left(-\mu^{2}\left(\sqrt{2} x_{c}+\alpha\left(-4 \zeta \xi+\chi_{0}\right)\right)\right)}{\alpha}, \\
r_{7}=2 F 1\left[1,1+\frac{i k \alpha}{\sqrt{2} \mu^{2}}, 2+\frac{i k \alpha}{\sqrt{2} \mu^{2}}, r_{6}\right]
\end{gathered}
$$

and $2 F 1$ is the Hypergeometric function.

From a detailed investigation of the dependence of $F^{2}(k)$ (Eq. (14)) on its parameters, we observed that DCE depends most strongly on the width $\alpha$ of the similariton. To study this dependence, we fixed the values of the other parameters as $x_{0}=1, \chi_{0}=0.3, \zeta_{0}=1, \epsilon=1, \mu=1, \xi=1$. In Fig. 1, we plot the modal fraction of the bright similariton solution for several values of $\alpha$, showing that all have a clear bell-shaped curve with a maximum at $k=0$.

The evolution of DCE for the bright similariton (given by Eq. (12)) as a function of the time parameter $\zeta(t)$ was obtained using Eq. (44) for several values of $\alpha$. As shown in Fig. 2, DCE saturates in all cases at a minimum value sensitive to the value of $\alpha$. For $\alpha=0.1,0.5,1.0,1.5$ the saturation occurs at $\zeta(t) \simeq 0.001,0.02,0.1,0.2$, respectively: the smaller the width $\alpha$, the earlier the similariton reaches the saturation value. The same behavior has been observed for all $\alpha$ belonging to $[0.1,1.5]$. 
(a)

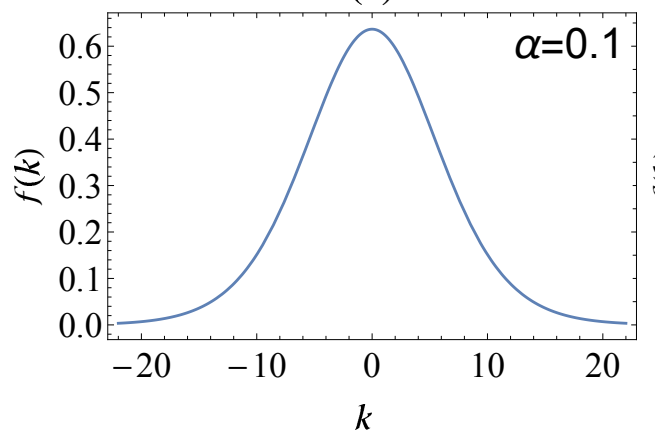

(c)

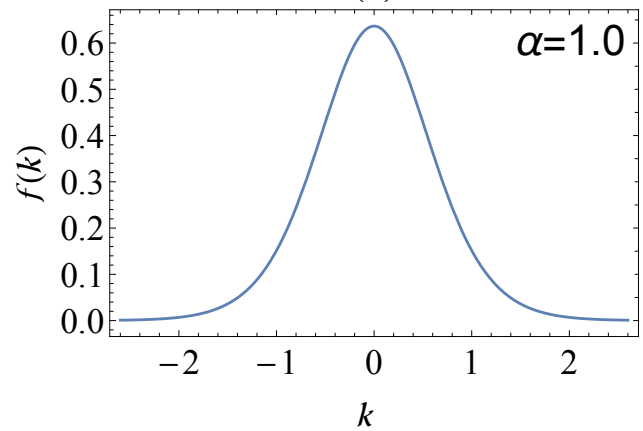

(b)

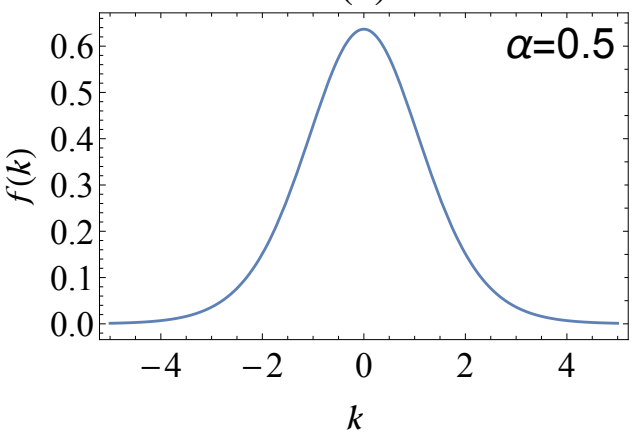

(d)

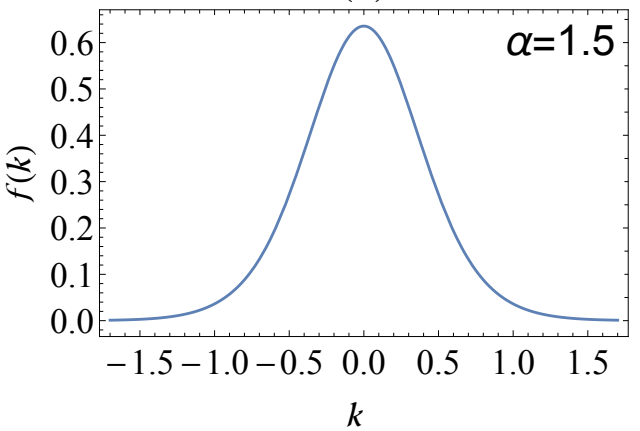

Figure 1: The modal fractions for bright similaritons with $\alpha=0.1,0.5,1.0,1.5$ showing a maximum at $k=0$. 
(a)

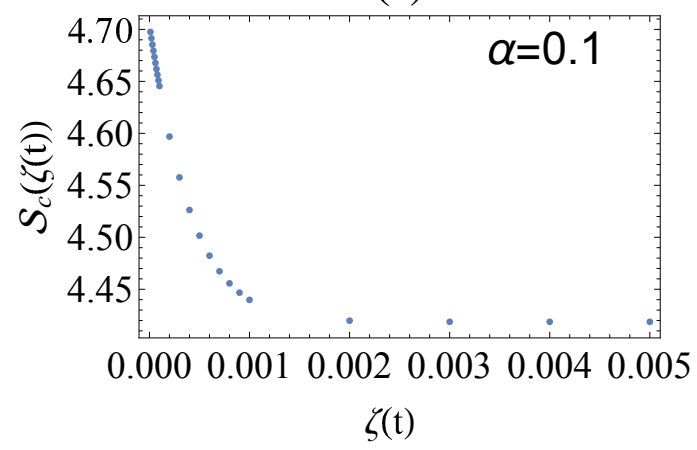

(c)

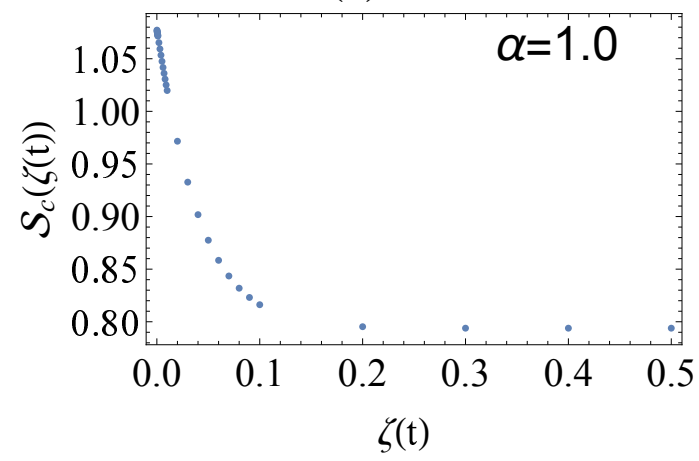

(b)

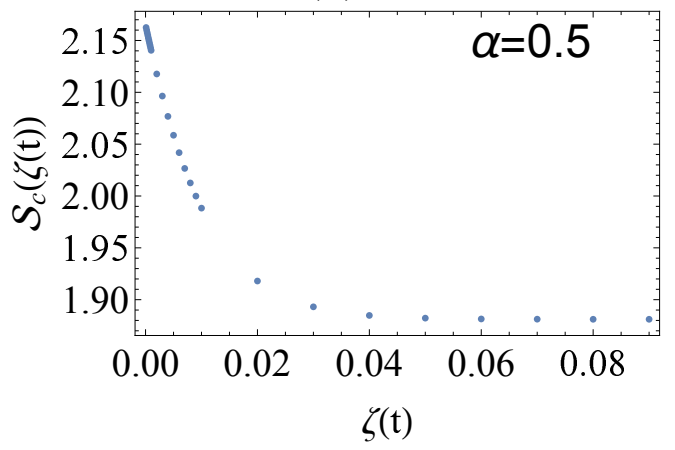

(d)

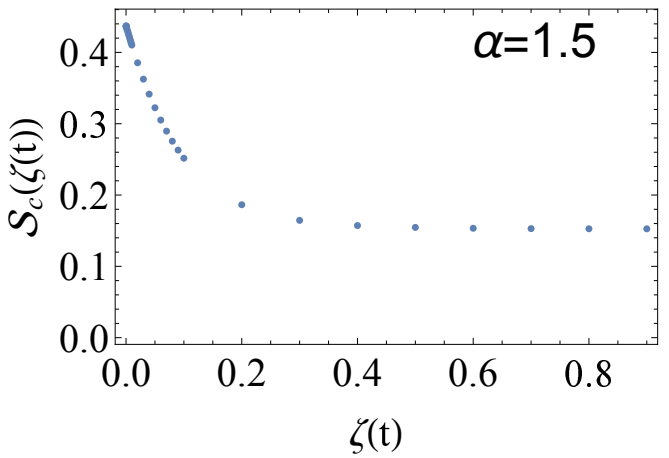

Figure 2: Differential Configurational entropy of bright optical similariton in the tapered graded-index waveguide propagating in the $\zeta(t)$ time at similariton width (a) $\alpha=0.1$, (b) $\alpha=0.5$, (c) $\alpha=1.0$, (d) $\alpha=1.5$.

Alternatively, one may examine the dependence of $S_{c}^{\alpha}(\zeta(t))$ on the width parameter $\alpha$ itself at different fixed times in the evolution of the bright similariton. The results are shown in Fig. 3 for three snapshots of $\zeta(t)=$ $0.1,0.3,0.5$. Overall, we find that $S_{c}^{\alpha}(\zeta(t))$ is weakly dependent on $\zeta(t)$, with a global minimum at $\alpha \simeq 1.8,1.7,1.6$, respectively. Furthermore, comparing Figs. 2 and Fig. 3 we note that the saturation values for different $\alpha$ in Fig. 2 correspond to the values of $S_{c}^{\alpha}(\zeta(t))$ in Fig. 3, This can be clearly seen comparing Figs. 2(d) and 3(c), since the values of $\alpha$ for both are near the global minimum of $S_{c}^{\alpha}(\zeta(t))$. As discussed in the literature (see e.g. [16, 17, 18, 23, 25, 28]), a minimum of DCE signals the most stable configuration with respect to a given parameter, in this case the similariton width 
(a)

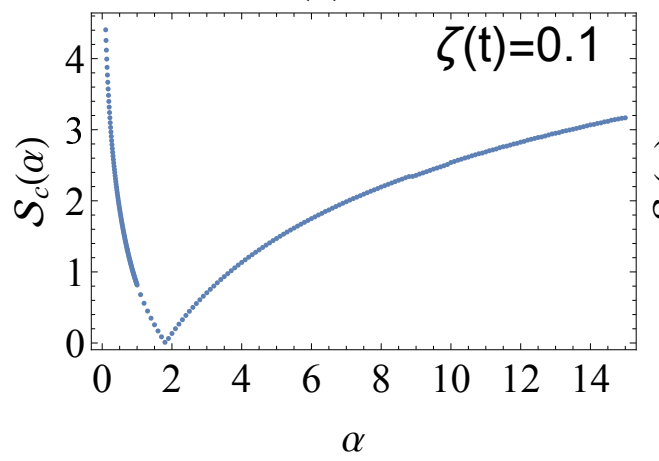

(b)

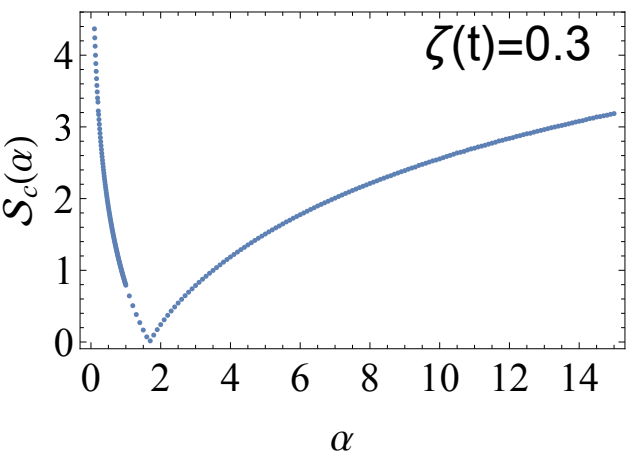

(c)

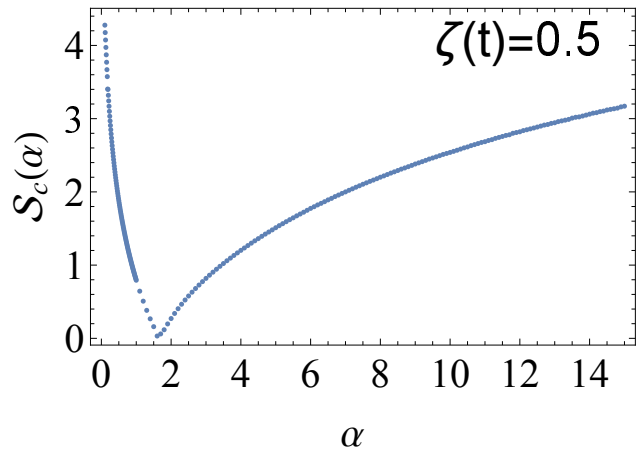

Figure 3: Differential configurational entropy $\left(S_{c}(\alpha)\right)$ as a function of the similariton width $\alpha$. The minimum value of $\operatorname{DCE}\left(S_{c}(\alpha)\right)$ occurs at (a) $\alpha=1.8$, (b) $\alpha=1.7$, (c) $\alpha=1.6$.

$\alpha$. These values denote the best range for the similariton's width to ensure its propagation through the tapered graded-index waveguide with optimal compression of information.

\section{Conclusion}

The tapered graded-index waveguide finds applications in optical fiber communications [32], computer networks [33], long-distance telecommunications [34], and sensory receptor cells [35, 43]. In this work, we have computed the differential configurational entropy $\left(S_{c}^{\alpha}(\zeta(t))\right.$ for optical bright similaritons propagating along a tapered graded-index waveguide. We found that 
$S_{c}^{\alpha}(\zeta(t))$ has a weakly time-dependent global minimum for a narrow range of width $\alpha$. We can thus see that this formalism helps one obtain the optimal width of the similariton waves for which dispersion is minimized and the spatial shape is most compressed into its momentum modes. This optimal compression ensures the propagation of minimally-entropic similariton waves through the tapered graded-index waveguide. In future work, we may investigate the dependence of our results on different values of the other similariton parameters and expand our method to two and three spatial dimensions, computing the related differential configurational entropy (DCE) [23]. We

hope this work will aid in the design of more efficient tapered graded-index waveguides with optimized widths.

\section{Acknowledgment}

The financial support from Department of Science and Technology, New Delhi through Women Scientist Scheme-A project (Ref. No. SR/ WOSA/ PM-109/2017 G) is gratefully acknowledged by Pooja Thakur. 


\section{References}

[1] R. Rajaraman, Solitons and Instantons, North-Holland, Amsterdam, (1987).

[2] R. K. Dodd, J. C. Eilbeck, J. D. Gibbon, and H. C. Morris, Solitons and Nonlinear Wave Equation, Academic Press, New York, (1982).

[3] J. S. Russell, Fourteenth meeting of the British Association for the Advancement of Science, 14311 (1844).

[4] M. Wadati, Pramana-J. Phys, 57841 (2001).

[5] A. M. Dudarev, R. B. Diener, and Q. Niu, J. Opt. B: Quant. Semiclass. Opt., 6 S231 (2004).

[6] D. N. Christodoulides and R. I. Joseph, Opt. Lett., 13794 (2004).

[7] W. P. Su, J. R. Schieffer, and A. J. Heeger, Phys. Rev. Lett., 421968 (1979).

[8] A. J. Sievers and S. Takeno, Phys. Rev. Lett., 61970 (1988).

[9] A. Trombettoni and A. Smerzi, Phys. Rev. Lett., 862353 (2001).

[10] P. Painleve, Acta Math., 251 (1902).

[11] M. Lakshmanan and S. Rajaseekar, Nonlinear Dynamics, Springer, (2003).

[12] Q. H. Ansari, C. S. Lalitha, and M. Mehta, Taylor and Francis, Generalized Convexity, Nonsmooth Variational Inequalities, and Nonsmooth Optimization, (2014).

[13] C. E. Shannon and W. Weaver, The Mathematical Theory of Communication, University of Illinois Press, Urbana, (1949).

[14] M. Gleiser and N. Stamatopoulos, Phys. Lett. B, 713304 (2012). arXiv:1111.5597.

[15] M. Gleiser and N. Stamatopoulos, Phys. Rev. D, 86045004 (2012). arXiv:1205.3061 
[16] M. Gleiser and N. Jiang, Phys. Rev. D, 92044046 (2015).

[17] A. E. Bernardini, N. R. F. Braga, and R. da Rocha, Phys. Lett. B, 765 81 (2017).

[18] M. Gleiser and D. Sowinski, Phys. Lett. B, 727217 (2013).

[19] N. R. F. Braga and R. da Rocha, Phys. Lett. B, 767386 (2017).

[20] C. O. Lee, Phys. Lett. B, 772471 (2017).

[21] R. Casadio and R. da Rocha, Phys. Lett. B, 763434 (2016).

[22] G. Karapetyan, Eur. Phys. Lett., 12558001 (2019).

[23] M. Gleiser, M. Stephens, and D. Sowinski, Phys. Rev. D, 97096007 (2018).

[24] M. Gleiser and D. Sowinski, Phys. Rev. D, 98056026 (2018).

[25] N. B. Cendjas, R. C. Fuentevilla, A. H. Aguilar, R. R. M. Luna, and R. da Rocha, Phys. Lett. B, 782607 (2018).

[26] A. E. Bernardini and R. da Rocha, Phys. Lett. B, 796107 (2019).

[27] M. Gleiser and N. Graham, Phys. Rev. D, 89, 083502 (2014). arXiv:1401.6225

[28] N. R. F. Braga, L. F. Ferreira, and R. da Rocha, Phys. Lett. B, 78716 (2018).

[29] M. Gleiser and D. Sowinski, Phys. Lett. B, 772471 (2015).

[30] R. A. Correa, A. de Souza Dutra, and M. Gleiser, Phys. Lett. B, 737 388 (2014).

[31] A. Goncalves da Silva and R. da Rocha, Phys. Lett. B, 77498 (2017).

[32] J. Arrue, F. Jimenez, G. Aldabaldetreku, G. Durana, and J. Zubia, J. Opt. Soc. Am., 1616616 (2008).

[33] B. Sengupta, S. B. Laughlin, and J. E. Niven, Comput. Biol., 10(1) e1003439 (2014). 
[34] Q. Bi and K. Song, J. Mod. Phys., 4923 (2013).

[35] P. Liu, B. Chen, and Z. W. Wang, Nat. Commun., 41 (2013).

[36] G. P. Agrawal, Nonlinear Fiber Optics, Academic Press, San Diego, (2001).

[37] S. A. Ponomarenko and G. P. Agrawal, Opt. Lett., 321659 (2007).

[38] C. N. Kumar, R. Gupta, A. Goyal, and S. Loomba, Phys. Rev. A, 86 025802 (2012).

[39] C. E. Shannon, Bell. Syst. Tech. J., 27379 (1948).

[40] V. E Zakharov and A. B. Shabat, Sov. Phys. JETP, 34118 (1971).

[41] V. E Zakharov and A. B. Shabat, Sov. Phys. JETP, 37823 (1973).

[42] N. Akhmediev, A. Ankiewicz, and M. Taki, Phys. Lett. A, 373675 (2009).

[43] M. Juusola, A. S. French, R. O. Uusitalo, and M. Weckström, TRENDS NEUROSCI, 197 (1996). 